\title{
Effect of Phototherapy on the Reliability of Transcutaneous Bilirubin Devices in Term and Near-Term Infants: A Systematic Review and Meta-Analysis
}

\author{
Gaurav Nagar $^{\mathrm{a}}$ Ben Vandermeer $^{\mathrm{b}}$ Sandy Campbell ${ }^{\mathrm{c}}$ Manoj Kumar ${ }^{\mathrm{a}}$ \\ ${ }^{a}$ Division of Neonatology, Department of Pediatrics, ${ }^{b}$ Alberta Research Center for Health Evidence, and \\ 'John W. Scott Health Sciences Library, University of Alberta, Edmonton, Alta., Canada
}

\section{Key Words}

Transcutaneous bilirubin - Correlation coefficient .

Bland-Altman difference plots - Systematic review .

Meta-analysis

\begin{abstract}
Background: Transcutaneous bilirubin (TcB) devices are commonly used for screening of hyperbilirubinemia in term and near-term infants not exposed to phototherapy. However, the accuracy of TcB devices in infants exposed to phototherapy is unclear. Objectives: To conduct a systematic review of studies comparing TCB devices with total serum bilirubin (TSB) in infants receiving phototherapy or in the postphototherapy phase. Methods: MEDLINE, EMBASE, Cochrane Library, CINAHL and Scopus databases (from inception to May 8, 2014) were searched. Additional citations were identified from the bibliography of selected articles and from the abstracts of conference proceedings. The studies were included if they compared TCB results with TSB in term and near-term infants during phototherapy or after discontinuation of phototherapy. Two reviewers independently assessed studies for inclusion, and discrepancies were resolved with consensus. Risk of bias was assessed using the
\end{abstract}

QUADAS-2 tool. Results: Fourteen studies were identified. The pooled estimates of correlation coefficients ( $r$ ) during phototherapy were: covered sites $0.71(95 \% \mathrm{Cl} 0.64-0.77,11$ studies), uncovered sites 0.65 ( $95 \% \mathrm{Cl} 0.55-0.74)$, 8 studies), forehead 0.70 ( $95 \% \mathrm{Cl} 0.64-0.75,12$ studies) and sternum 0.64 ( $95 \% \mathrm{Cl} 0.43-0.77,5$ studies). Two studies also provided results as Bland-Altman difference plots (mean TcB-TSB differences -29.2 and $30 \mu \mathrm{mol} / \mathrm{l}$, respectively). The correlation coefficient improved marginally in the postphototherapy phase ( $r=0.72,95 \% \mathrm{Cl} 0.64-0.78,4$ studies). Conclusion: We found a moderate correlation between TCB and TSB during phototherapy with a marginal improvement in the postphototherapy phase. Further research is needed before the use of TCB devices can be recommended for these settings.

(c) 2016 S. Karger AG, Basel

\section{Introduction}

Jaundice is common in the newborn period with the majority of neonates developing visible jaundice within the first week of life $[1,2]$. Although in a significant majority this does not possess any risk to the newborn, up to $10 \%$ of term and $25 \%$ of near-term neonates develop sig-

\section{KARGER}

E-Mail karger@karger.com

www.karger.com/neo
(C) 2016 S. Karger AG, Basel

$1661-7800 / 16 / 1093-0203 \$ 39.50 / 0$
Manoj Kumar, MD, MSc (Clinical Epidemiology)

Department of Pediatrics, Edmonton Clinical Health Academy

Room 3-528, 1140587 Avenue NW

Edmonton, AB T6G 1C9 (Canada)

E-Mailmanojk@ualberta.ca 
nificant hyperbilirubinemia necessitating phototherapy [3]. Newborn jaundice is known to have cephalocaudal progression [4]; however, visual assessment of serum bilirubin has been shown to correlate poorly with measured bilirubin levels in recent studies $[2,5,6]$. Blood sampling for the estimation of serum bilirubin is one of the commonest tests ordered in the neonatal units. This is often done by heel prick and is considered painful, with potential long-term consequences [7].

Transcutaneous bilirubin $(\mathrm{TcB})$ devices estimate serum bilirubin noninvasively from the skin [8]. These devices have been shown to correlate well with serum bilirubin levels in term and preterm infants prior to the start of phototherapy [9-14]. The American Academy of Pediatrics recommends the use of $\mathrm{TcB}$ devices for the screening of jaundice in infants at more than 35 weeks of gestation [15].

Infants requiring phototherapy need frequent measurement of serum bilirubin to assess the response to treatment. However, the role of $\mathrm{TcB}$ devices during phototherapy or in the postphototherapy phase is not clear. Recently there has been an increasing trend towards home phototherapy in the low-risk term and near-term infants [16-19], where arranging follow-up assessments of serum bilirubin is cumbersome. Such infants could also benefit from assessment by a TcB device, if validated.

The objective of this systematic review was to assess the diagnostic accuracy of transcutaneous devices as compared to the total serum bilirubin (TSB) measurement in term and near-term infants receiving phototherapy during the neonatal period.

\section{Methods}

Search and Selection

Several databases including MEDLINE, EMBASE, Cochrane Library, CINAHL and Scopus were systematically searched (initially searched in August 2013 and search updated on May 8, 2014). Both controlled vocabulary terms (e.g. MeSH, EMTREE, etc.) and key words were used to search the concepts: neonatal, bilirubinometers, transcutaneous, total serum bilirubin and phototherapy. Additional terminology and predefined database limits were added to restrict the references to those related to less than 1 month of age. No language restriction was applied (refer to the Appendix for the detailed search strategy). Conference proceedings and bibliography of included studies were also searched for any potential studies.

Studies were included in the review if they enrolled infants $\geq 34$ weeks of gestational age and compared TcB results to TSB estimation during the neonatal period while receiving phototherapy. We excluded pilot studies (defined a priori as those enrolling $\leq 20$ subjects) and studies enrolling infants of all gestational ages but not providing separate data for the population of interest. The out- come of interest was agreement statistic between TcB and TSB measurements provided either as correlation coefficient or as the mean and standard deviations of absolute differences in bilirubin values by the two methods (Bland-Altman difference plots) [20].

\section{Data Extraction and Assessment of Risk of Bias}

Titles, abstracts and citations were independently assessed by two reviewers for inclusion based on predefined selection criteria. Data from included studies were extracted on a specifically designed data extraction form by one reviewer and checked for accuracy by a second reviewer. Risk of bias assessments were conducted according to the QUADAS-2 (Quality Assessment of Diagnostic Accuracy Studies) tool [21]. This tool consists of 4 key domains: patient selection, index test, reference standard and flow and timing. Each study is assessed for risk of bias in each of the domains and for concerns regarding applicability in the first 3 domains. Disagreements were resolved by consensus among the members of the review team.

\section{Data Analysis}

We meta-analyzed the available data both from the correlation coefficients of the measurements of TcB and TSB, and from the Bland-Altman difference plots. All correlations were first converted to Fisher $\mathrm{Z}$ scores prior to being pooled. The resulting pooled Fisher $\mathrm{Z}$ scores were then transformed back into standard correlation coefficients for the ease of interpretation. For Bland-Altman difference plots, we pooled the mean TcB-TSB differences and variance across eligible studies for estimation of bias and standard deviation, respectively, by methods as described by Peyton and Chong [22].

A priori subgroup analyses were planned to explore the influence of the shielded area, site of TcB measurement and the type of $\mathrm{TcB}$ device used. We also assessed the accuracy of the TcB devices in the postphototherapy phase where studies provided those data.

Meta-analyses were performed using Review Manager version 5.2 software (Nordic Cochrane Centre, Cochrane Collaboration, 2011, Copenhagen, Denmark). The $\mathrm{I}^{2}$ statistic was calculated for each analysis to quantify heterogeneity across studies. Forest plots were created using SPlus Software version 3.4 (TIBCO Software Inc., 2010).

\section{Results}

We identified 14 studies [17, 23-35] providing 2,082 paired measurements of TcB and TSB in 1,319 patients who fulfilled the inclusion criteria (fig. 1). The baseline characteristics of the included studies are presented in table 1. Studies varied in terms of: whether the site(s) of measurement were shielded from phototherapy; the actual site of $\mathrm{TcB}$ measurement (forehead $=12$ studies; sternum $=5$ studies and other sites $=2$ studies); the $\mathrm{TcB}$ device used (Bilicheck = 1 study; JM 103 = 3 studies; JM $101=10$ studies); the method used for serum bilirubin measurement (direct spectrophotometry only $=7$ studies; diazo method only $=5$ studies; both $=1$ study, and meth- 
Fig. 1. Flow of studies through the selection process.

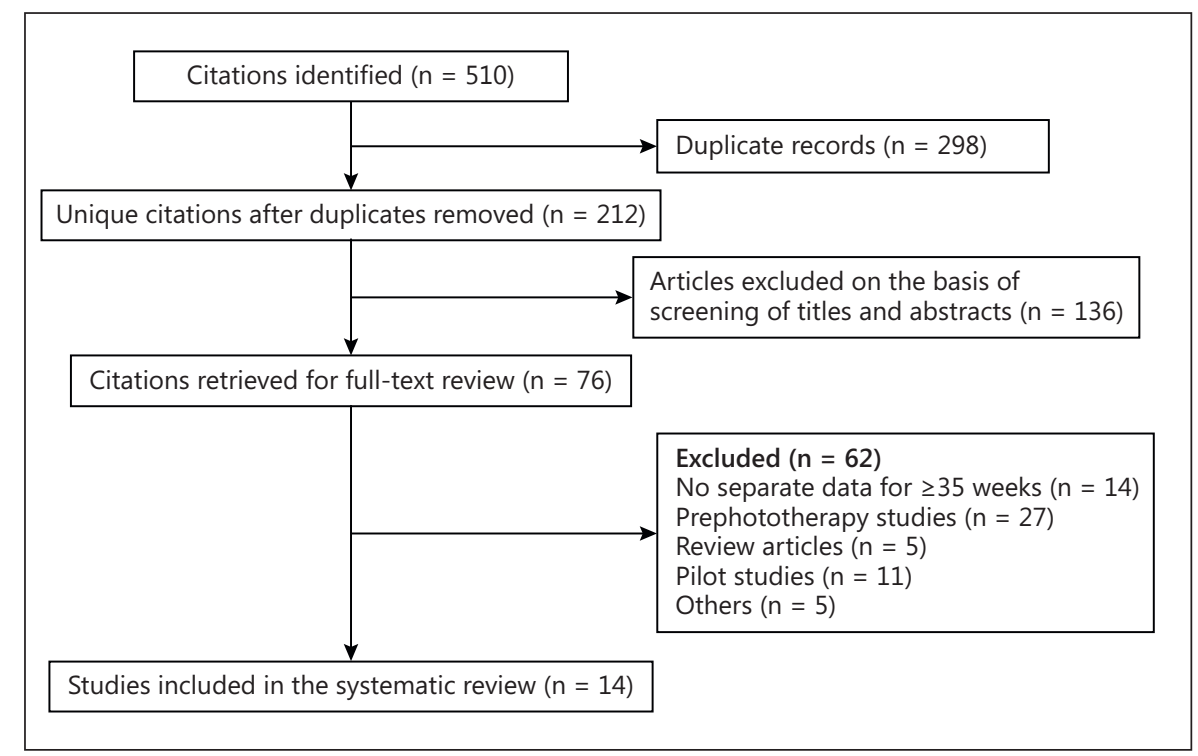

od not specified = 1 study), and agreement statistic used for comparison (correlation coefficient $=14$ studies $[17$, 23-35], Bland-Altman difference plots $=2$ studies [17, 23]; 2 studies reported results with both methods). Four studies $[27,32,34,35]$ provided data for the postphototherapy phase, as correlation coefficients.

Results for risk of bias assessments for the included studies are provided in table 2 . The majority of the included studies were assessed as low risk for bias with respect to patient selection, index test, reference standard and flow timing. However, there were some applicability concerns with most of the included studies. Ten studies [24-33] employed TcB devices (Minolta Airshields Jaundice/Bilirubin Meter, i.e. JM 101) that are no longer used in clinical practice. In the study by Rylance et al. [23], although the blood for estimation of TSB was collected at the time of $\mathrm{TcB}$ estimation, samples were processed once a day resulting in greater time lag and thus risk of degradation of bilirubin from light and heat [36]. Nine studies $[17,23,25,26,29,30,32,33,35]$ conducted the TcB and TSB estimations simultaneously or within $30 \mathrm{~min}$, whereas the remaining $5[24,27,28,31,34]$ did not specify the time difference between the two tests.

Results of Meta-Analyses of Data from Subjects during Phototherapy (13 Studies)

Correlation Coefficients

All studies provided results for correlation coefficients. Separate meta-analyses were conducted for whether the site of measurement was shielded from direct photother- apy light (covered and uncovered areas; fig. 2) and for the two commonly used sites of measurement, i.e. the forehead and sternum (fig. 3).

The pooled estimate of the correlation coefficients from the 11 studies [23-30, 32, 33, 35] providing data from sites covered during phototherapy was 0.71 (95\% CI $0.64-0.77)$. The estimate from the uncovered sites was slightly lower ( $\mathrm{r}=0.65,95 \%$ CI $0.55-0.74$, in 8 studies) $[17,24,26,27,29-32]$; however, this difference was not statistically significant (fig. 2). Similarly, there was no statistically significant difference noted between the estimates from the newer TcB devices (JM 103 and Bilicheck) as compared to the older devices.

Results of the Pooled Estimates according to the Site of Measurement. Twelve studies provided data for the TcB measurements at the forehead site for comparison with TSB results, and the summated correlation coefficient from this site was 0.70 (95\% CI 0.64-0.75). The majority of the included studies covered the forehead site; however, 2 studies $[29,32]$ also provided data from an uncovered area over the forehead. The summated correlation coefficient was unchanged after removing those data $(\mathrm{r}=$ $0.70,95 \%$ CI $0.64-0.76$ ). The pooled estimate from the studies providing data for $\mathrm{TcB}$ measurements at the sternum (5 studies) was 0.64 (95\% CI $0.43-0.77)$. There was significant heterogeneity noted in this estimate $\left(\mathrm{I}^{2}=\right.$ $77 \%)$. 
Table 1. Characteristics of the included studies

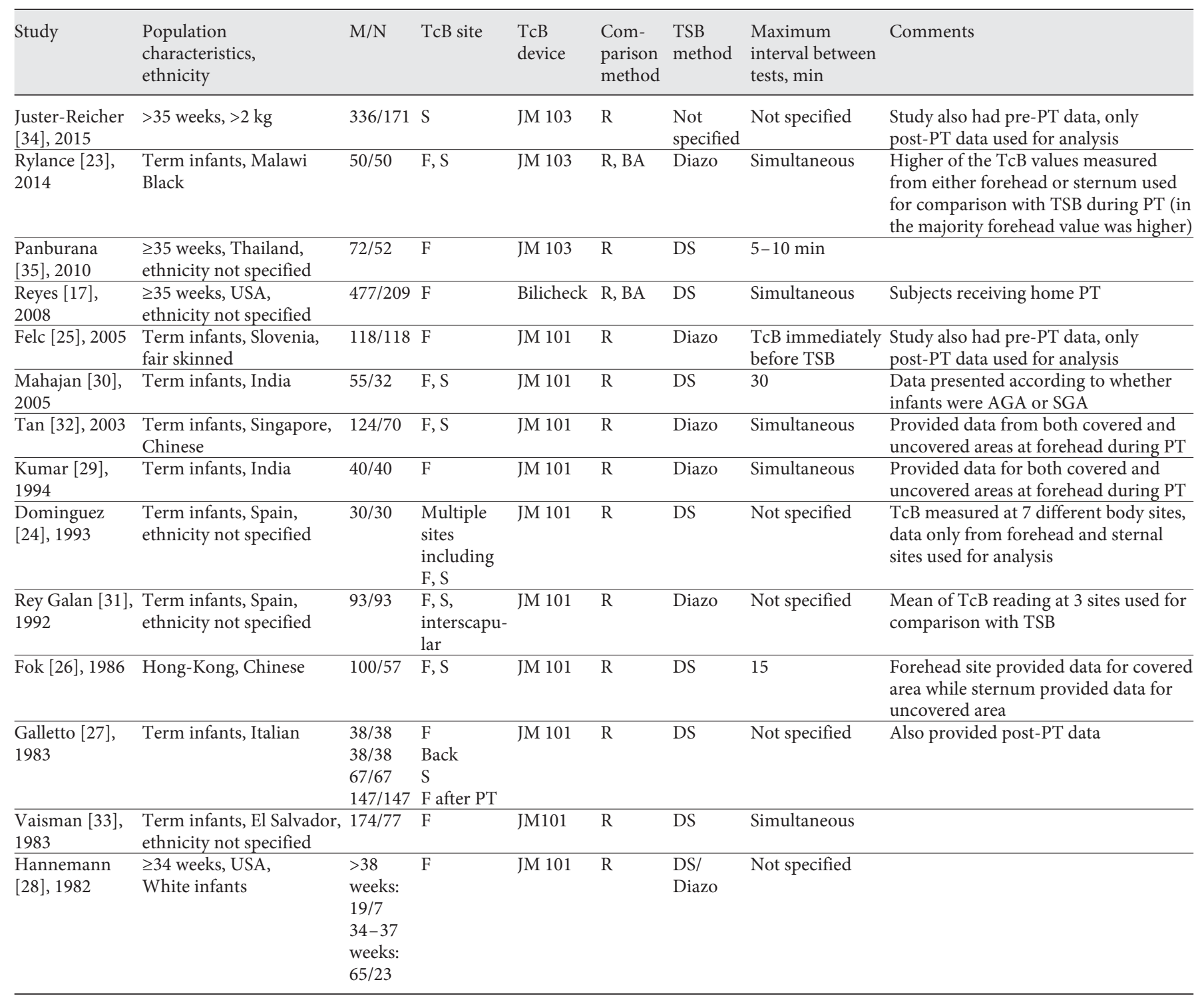

$\mathrm{M} / \mathrm{N}=$ Number of measurements/subjects; $\mathrm{F}$ = forehead; $\mathrm{S}=$ sternum; $\mathrm{PT}=$ phototherapy; JM 101 = referred as Minolta Air Shields Jaundice/Bilirubin Meter; $\mathrm{R}$ = correlation coefficient; $\mathrm{BA}=$ Bland-Altman difference plots; $\mathrm{DS}=$ direct spectrophotometry; $\mathrm{AGA}=$ appropriate for gestational age; $\mathrm{SGA}=$ small for gestational age.

Bland-Altman Difference Plots

Only 2 studies [17,23] provided data for Bland-Altman difference plots. The study by Reyes et al. [17] using the Bilicheck device at the forehead site showed that TcB underestimated TSB by a mean difference of $29.2 \mu \mathrm{mol} / \mathrm{l}$. On the other hand, the study by Rylance et al. [23] using the JM 103 device showed that TcB overestimated TSB (mean difference $=30 \mu \mathrm{mol} / \mathrm{l}, 95 \%$ precision limits $\pm 79 \mu \mathrm{mol} / \mathrm{l})$.
TcB-TSB Comparison in Postphototherapy Phase

(4 Studies)

Four of the included studies $[27,32,34,35]$ also provided data for infants in the postphototherapy phase (fig. 4). The correlation between TcB and TSB readings improved marginally following discontinuation of phototherapy ( $\mathrm{r}=0.72,95 \%$ CI $0.64-0.78)$. Tan and Dong [32] noted further improvement in the correlation coef- 
Table 2. Risk of bias assessments of the included studies

\begin{tabular}{|c|c|c|c|c|c|c|c|}
\hline \multirow[t]{2}{*}{ Study } & \multicolumn{4}{|c|}{ Risk of bias } & \multicolumn{3}{|c|}{ Applicability concerns } \\
\hline & $\begin{array}{l}\text { patient } \\
\text { selection }\end{array}$ & $\begin{array}{l}\text { index } \\
\text { test }\end{array}$ & $\begin{array}{l}\text { reference } \\
\text { standard }\end{array}$ & $\begin{array}{l}\text { flow and } \\
\text { timing }\end{array}$ & $\begin{array}{l}\text { patient } \\
\text { selection }\end{array}$ & index test & $\begin{array}{l}\text { reference } \\
\text { standard }\end{array}$ \\
\hline Juster-Reicher [34], 2015 & $\checkmark$ & $\checkmark$ & $\checkmark$ & $?$ & $\checkmark$ & $\checkmark$ & $?$ \\
\hline Rylance [23], 2014 & $\checkmark$ & $\checkmark$ & $\checkmark$ & $\checkmark$ & $\checkmark$ & $\checkmark$ & $?$ \\
\hline Panburana [35], 2010 & $\checkmark$ & $\checkmark$ & $\checkmark$ & $\checkmark$ & $\checkmark$ & $?$ & $\checkmark$ \\
\hline Reyes [17], 2008 & $\checkmark$ & $\checkmark$ & $\checkmark$ & $\checkmark$ & $\checkmark$ & $\checkmark$ & $\checkmark$ \\
\hline Felc [25], 2005 & $\checkmark$ & $\checkmark$ & $\checkmark$ & $\checkmark$ & $\checkmark$ & $?$ & $\checkmark$ \\
\hline Mahajan [30], 2005 & $\checkmark$ & $\checkmark$ & $\checkmark$ & $\checkmark$ & $\checkmark$ & $?$ & $\checkmark$ \\
\hline Tan [32], 2003 & $\checkmark$ & $\checkmark$ & $\checkmark$ & $\checkmark$ & $\checkmark$ & $?$ & $\checkmark$ \\
\hline Kumar [29], 1994 & $\checkmark$ & $\checkmark$ & $\checkmark$ & $\checkmark$ & $\checkmark$ & $?$ & $\checkmark$ \\
\hline Dominguez [24], 1993 & $\checkmark$ & $\checkmark$ & $\checkmark$ & $?$ & $\checkmark$ & $?$ & $\checkmark$ \\
\hline Rey Galan [31], 1992 & $\checkmark$ & $\checkmark$ & $\checkmark$ & $?$ & $\checkmark$ & $?$ & $\checkmark$ \\
\hline Fok [26], 1986 & $\checkmark$ & $\checkmark$ & $\checkmark$ & $\checkmark$ & $\checkmark$ & $?$ & $\checkmark$ \\
\hline Galletto [27], 1983 & $?$ & $\checkmark$ & $\checkmark$ & $?$ & $\checkmark$ & $?$ & $\checkmark$ \\
\hline Vaisman [33], 1983 & $\checkmark$ & $\checkmark$ & $\checkmark$ & $\checkmark$ & $\checkmark$ & $?$ & $\checkmark$ \\
\hline Hannemann [28], 1982 & $\checkmark$ & $\checkmark$ & $\checkmark$ & $?$ & $\checkmark$ & $?$ & $?$ \\
\hline
\end{tabular}

$\checkmark=$ Low risk; ? = unclear risk.

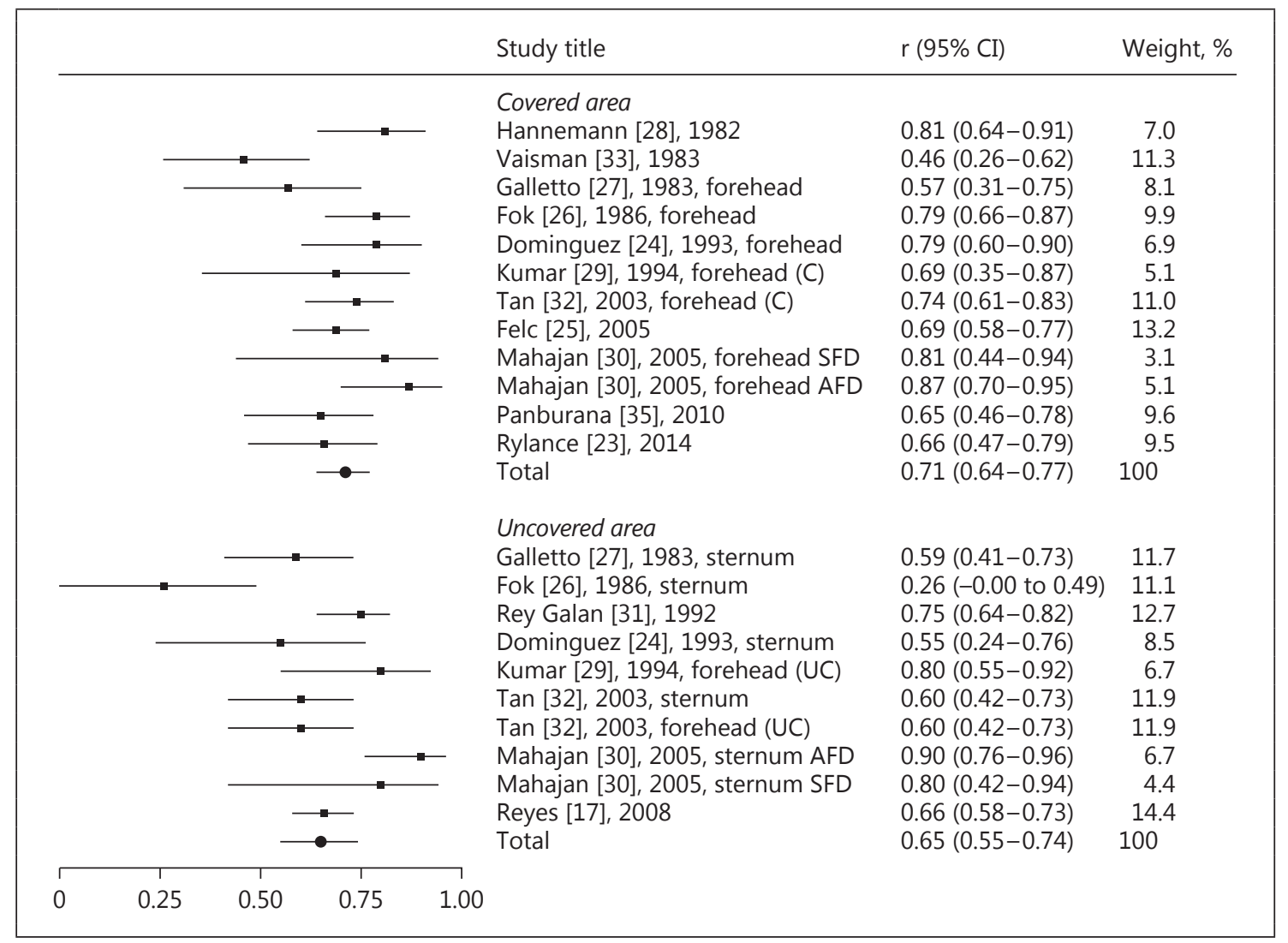

Fig. 2. Pooled estimates of correlation coefficients at covered and uncovered sites during phototherapy. $C=T c B$ data from 'covered' area over forehead; $\mathrm{UC}=\mathrm{TcB}$ data from 'uncovered' area over forehead; SFD = small-fordates subset; AFD = appropriate-for-dates subset.

Reliability of Transcutaneous Bilirubin Devices
Neonatology 2016;109:203-212 DOI: $10.1159 / 000442195$ 


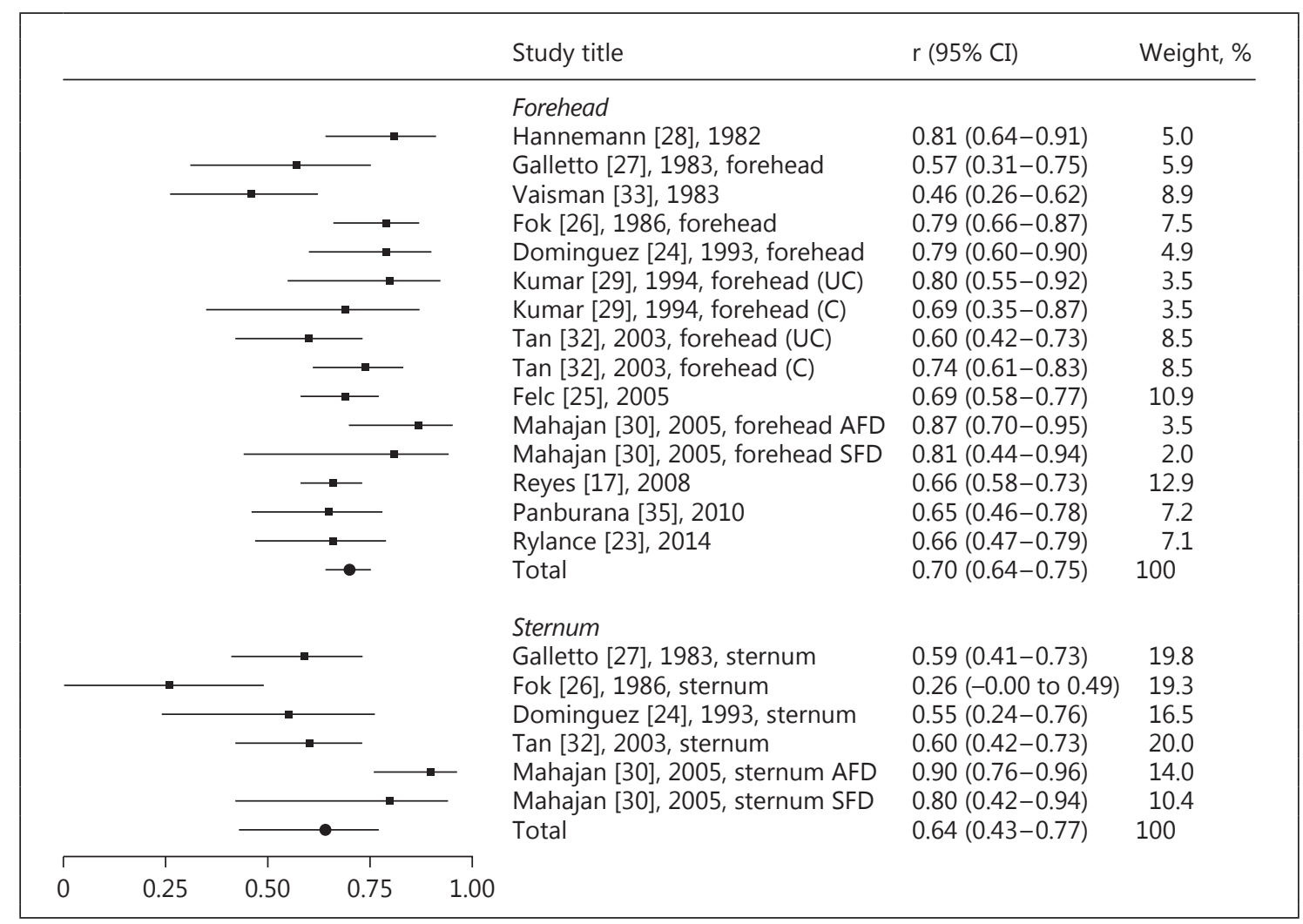

Fig. 3. Pooled estimates of correlation coefficients at the forehead and sternum during phototherapy. For abbreviations, see figure 2 .

\begin{tabular}{|c|c|c|c|c|c|c|}
\hline & & & & Study title & $r(95 \% \mathrm{CI})$ & Weight, \% \\
\hline & & & $\Longrightarrow$ & Galletto [27], 1983, post-PT & $0.71(0.62-0.78)$ & 24.7 \\
\hline & & & $\longrightarrow$ & Tan [32], 2003, post-PT S & $0.81(0.71-0.88)$ & 18.2 \\
\hline & & & $\longrightarrow$ & Tan [32], 2003, post-PT F & $0.76(0.64-0.85)$ & 18.2 \\
\hline & & & - & Panburana [35], 2010 & $0.47(0.18-0.68)$ & 12.8 \\
\hline & & & $\longrightarrow-$ & Juster-Reicher [34], 2015 & $0.72(0.64-0.79)$ & 26.0 \\
\hline & & & $\longrightarrow$ & Total & $0.72(0.64-0.78)$ & 100 \\
\hline$\Gamma$ & 1 & 1 & 1 & ᄀ & & \\
\hline 0 & 0.25 & 0.50 & 0.75 & 1.00 & & \\
\hline
\end{tabular}

Fig. 4. Pooled estimates of correlation coefficients in the postphototherapy phase (PT). $S=$ Sternum; $F=$ forehead.

ficients on the second day after phototherapy as compared to the first reading taken $18-24 \mathrm{~h}$ after discontinuation of phototherapy (sternum: $\mathrm{r}=0.84$ on day 2 vs. 0.80 for day 1 ; forehead: $r=0.80$ on day 2 vs. 0.70 for day 1$)$.

\section{Discussion}

We identified 14 studies evaluating the diagnostic accuracy of various $\mathrm{TcB}$ devices in term and near-term infants after the onset of phototherapy. The results of this review show a moderate correlation [37] between the TcB 
and the TSB estimation in infants while receiving phototherapy, with a slightly better correlation noted at the forehead site or when the site of measurement was shielded from phototherapy light. The limited data available from the 4 studies assessing agreement between TcB and TSB estimation following discontinuation of phototherapy indicate a somewhat better agreement in the postphototherapy phase.

Whilst all studies provided correlation coefficients, only 2 studies provided data for the Bland-Altman difference plots. Unfortunately, the translation into clinical practice of a moderate correlation coefficient noted between TcB and TSB tests is difficult, as it does not provide information in terms of the expected difference between these measurements for an individual patient. On the other hand, data presented as Bland-Altman difference plots (in terms of bias and precision estimates) could allow a reasonable prediction of the range of the TSB values likely for a given $\mathrm{TcB}$ reading, which is thus much more helpful for clinical decision-making [20]. The limited data from the 2 studies providing Bland-Altman difference plots prevents us from making any firm recommendations for practice.

The pooled estimates of correlation coefficients noted here are much weaker than the estimates noted in several studies of term and near-term infants prior to subjects receiving phototherapy [9-13], where the correlation was noted to be high (i.e. coefficients typically ranged between 0.85 and 0.94 ) and the use of the TcB devices is recommended for decreasing blood sampling during screening for neonatal jaundice $[15,38,39]$. Based on the data presented here, the $\mathrm{TcB}$ devices cannot be relied upon for assessment of bilirubin in infants exposed to phototherapy. However, the improvement in TcB-TSB agreement noted in the postphototherapy phase seems to indicate that in future studies the accuracy of the TcB devices should be separately assessed in the period following discontinuation of phototherapy. Furthermore, the temporal changes in TcB-TSB agreement in the postphototherapy phase should also be studied, as suggested in one study [32].

Our review has a few limitations. First, the majority of the studies were done using older versions of $\mathrm{TcB}$ devices which are no longer in practice. It is possible that the current version of these devices may have better agreement with TSB. Also, one of the studies that assessed a TcB device used currently in practice [23] could be biased as blood samples for TSB estimation were stored for several hours before processing (processed once a day) leading to a significant risk of bilirubin degradation due to environ-

Reliability of Transcutaneous Bilirubin Devices mental factors. Second, we decided a priori to exclude studies enrolling $<20$ participants, as data from smaller studies are known to often distort results of the metaanalyses due to many reasons [40]. Third, the majority of the included studies presented results as correlation coefficients which are difficult to translate into clinical practice as discussed above. Fourth, we could not apply a formal test to check for publication bias due to a limited number of studies; however, the funnel plot of the included studies did not reveal any obvious asymmetry. Lastly, several of the studies included in the meta-analysis provided multiple readings from each patient enrolled, leading to the statistical risks of dependency of data. However, the results from the studies providing one reading per patient enrolled were not significantly different from those providing multiple data points per patient.

\section{Conclusion}

The results of this systematic review show that the TcB devices are much less accurate in estimating serum bilirubin values in infants when receiving phototherapy as compared to their documented accuracy in the prephototherapy period. The limited data available from the studies assessing agreement between TcB and TSB tests following discontinuation of phototherapy show a somewhat improved accuracy of the TcB devices in the postphototherapy phase. Further research with currently available TcB devices is needed before their use of TcB devices can be recommended in any of the settings that were the subject of this review.

\section{Acknowledgment}

We are grateful to Dr. Pharuhad Pongmee for his assistance with the identification of eligible studies for this systematic review.

\section{Disclosure Statement}

The authors do not have any financial disclosure to make.

Neonatology 2016;109:203-212

DOI: $10.1159 / 000442195$ 


\section{Appendix}

Bilirubinometers and Phototherapy Strategy

Database: Ovid MEDLINE ${ }^{\circledR}$ In-Process \& Other Non-Indexed Citations, Ovid MEDLINE ${ }^{\circledR}$

Daily and Ovid MEDLINE ${ }^{\circledR}<1946$ to present $>$

Search Strategy:

1 phototherapy.mp or exp Phototherapy/ $(29,242)$

2 (light adj2 therap*).mp [mp = title, abstract, original title, name of substance word, subject heading word, key word heading word, protocol supplementary concept, rare disease supplementary concept, unique identifier] $(1,668)$

3 phototherapies.mp (68)

4 photoradiation therap*.mp [ $\mathrm{mp}=$ title, abstract, original title, name of substance word, subject heading word, key word heading word, protocol supplementary concept, rare disease supplementary concept, unique identifier] (173)

51 or 2 or 3 or $4(29,965)$

6 (transcutaneous* and (bilirubin* or hyperbilirubin* or jaundice*)).mp [mp = title, abstract, original title, name of substance word, subject heading word, key word heading word, protocol supplementary concept, rare disease supplementary concept, unique identifier] (372)

7 tcb.mp [mp = title, abstract, original title, name of substance word, subject heading word, key word heading word, protocol supplementary concept, rare disease supplementary concept, unique identifier] (759)

8 (bilirubinometer* or biliblitz* or bilitest* or bilicheck* or bilichek* or tcbr or icterometer*). $\mathrm{mp}[\mathrm{mp}=$ title, abstract, original title, name of substance word, subject heading word, key word heading word, protocol supplementary concept, rare disease supplementary concept, unique identifier] (178)

96 or 7 or $8(1,066)$

105 and $9(126)$

Database: EMBASE <1974 to 2013 week 33>

Search Strategy:

1 phototherapy.mp or exp Phototherapy/ $(52,640)$

2 (light adj2 therap*).mp [mp = title, abstract, subject headings, heading word, drug trade name, original title, device manufacturer, drug manufacturer, device trade name, key word] $(2,478)$

3 phototherapies.mp (96)

4 photoradiation therap*.mp [mp = title, abstract, subject headings, heading word, drug trade name, original title, device manufacturer, drug manufacturer, device trade name, key word] (198)

51 or 2 or 3 or $4(53,514)$

6 (transcutaneous* and (bilirubin* or hyperbilirubin* or jaundice*)).mp [mp $=$ title, abstract, subject headings, heading word, drug trade name, original title, device manufacturer, drug manufacturer, device trade name, key word] (502)

7 tcb.mp [ $\mathrm{mp}=$ title, abstract, subject headings, heading word, drug trade name, original title, device manufacturer, drug manufacturer, device trade name, key word] (940)

8 (bilirubinometer* or biliblitz* or bilitest* or bilicheck* or bilichek* or tcbr or icterometer*). $\mathrm{mp}[\mathrm{mp}=$ title, abstract, subject headings, heading word, drug trade name, original title, device manufacturer, drug manufacturer, device trade name, key word] (231)

96 or 7 or $8(1,349)$

105 and $9(174)$

Ovid Technologies Inc. E-Mail Service

Search for: 5 and 9

Results: 1

Database: EBM Reviews - Cochrane Database of Systematic Reviews <2005 to July 2013>, EBM Reviews - ACP Journal Club $<1991$ to July 2013>, EBM Reviews - Database of Abstracts of Reviews of Effects $<3$ rd quarter 2013>, EBM Reviews - Cochrane Central Register of Controlled Trials <July 2013>, EBM Reviews - Cochrane Methodology Register <3rd quarter 2012>, EBM Reviews - Health Technology Assessment <3rd quarter 2013>, EBM Reviews NHS Economic Evaluation Database <3rd quarter 2013> 
Search Strategy:

1 phototherapy.mp or exp Phototherapy/ $(2,426)$

2 (light adj2 therap*).mp [mp = ti, ab, tx, kw, ct, ot, sh, hw] (452)

3 phototherapies.mp (19)

4 photoradiation therap*.mp [mp = ti, ab, tx, kw, ct, ot, sh, hw] (4)

51 or 2 or 3 or $4(2,631)$

6 (transcutaneous* and (bilirubin* or hyperbilirubin* or jaundice*)).mp [mp $=\mathrm{ti}, \mathrm{ab}, \mathrm{tx}, \mathrm{kw}$, ct, ot, sh, hw] (30)

7 tcb.mp [mp = ti, ab, tx, kw, ct, ot, sh, hw] (11)

8 (bilirubinometer* or biliblitz* or bilitest* or bilicheck* or bilichek* or tcbr or icterometer*). $\mathrm{mp}[\mathrm{mp}=\mathrm{ti}, \mathrm{ab}, \mathrm{tx}, \mathrm{kw}, \mathrm{ct}, \mathrm{ot}, \mathrm{sh}, \mathrm{hw}](12)$

96 or 7 or $8(40)$

105 and $9(15)$

$\mathrm{ti}=$ Title; $\mathrm{ab}=$ abstract $; \mathrm{tx}=$ full test $\mathrm{kw}=$ keywords; $\mathrm{ct}=$ caption text ot $=$ short title; $\mathrm{sh}=$ MeSH headings; hw = heading words.

Scopus

((TITLE-ABS-KEY(bilirubinometer* or biliblitz* or bilitest* or bilicheck* or bilichek* or tcbr or icterometer*) or (TITLE-ABS-KEY(transcutaneous* W/7 (bilirubin* or hyperbilirubin* or jaundice*))) ) and (TITLE-ABS-KEY(photo therap* or phototherap* or light therap* or light treatment* or photoradiat*))

ProQuest Dissertations and Theses Full Text, August 23, 2013

all(bilirubinometer* OR biliblitz* OR bilitest* OR bilicheck* OR bilichek* OR tcbr OR icterometer*) OR all(transcutaneous* NEAR/2 (bilirubin* OR hyperbilirubin* OR jaundice*)) AND all(photo therap* or phototherap* or light therap* or light treatment* or photoradiat*)

COMPENDEX and INSPEC searched together August 23, 2013

((photo NEAR/1 therap* OR phototherap OR light NEAR/1 therap* OR light NEAR/1 treatment* OR photoradiat*) AND (1985-2013 WN YR)) and ((((bilirubinometer* OR biliblitz* OR bilitest* OR bilicheck* OR bilichek* OR tcbr OR icterometer*) AND (1985-2013 WN YR)) OR ((transcutaneous* NEAR/2 bilirubin* OR transcutaneous* NEAR/2 hyperbilirubin* OR transcutaneous* NEAR/2 jaundice*) AND (1985-2013 WN YR))))

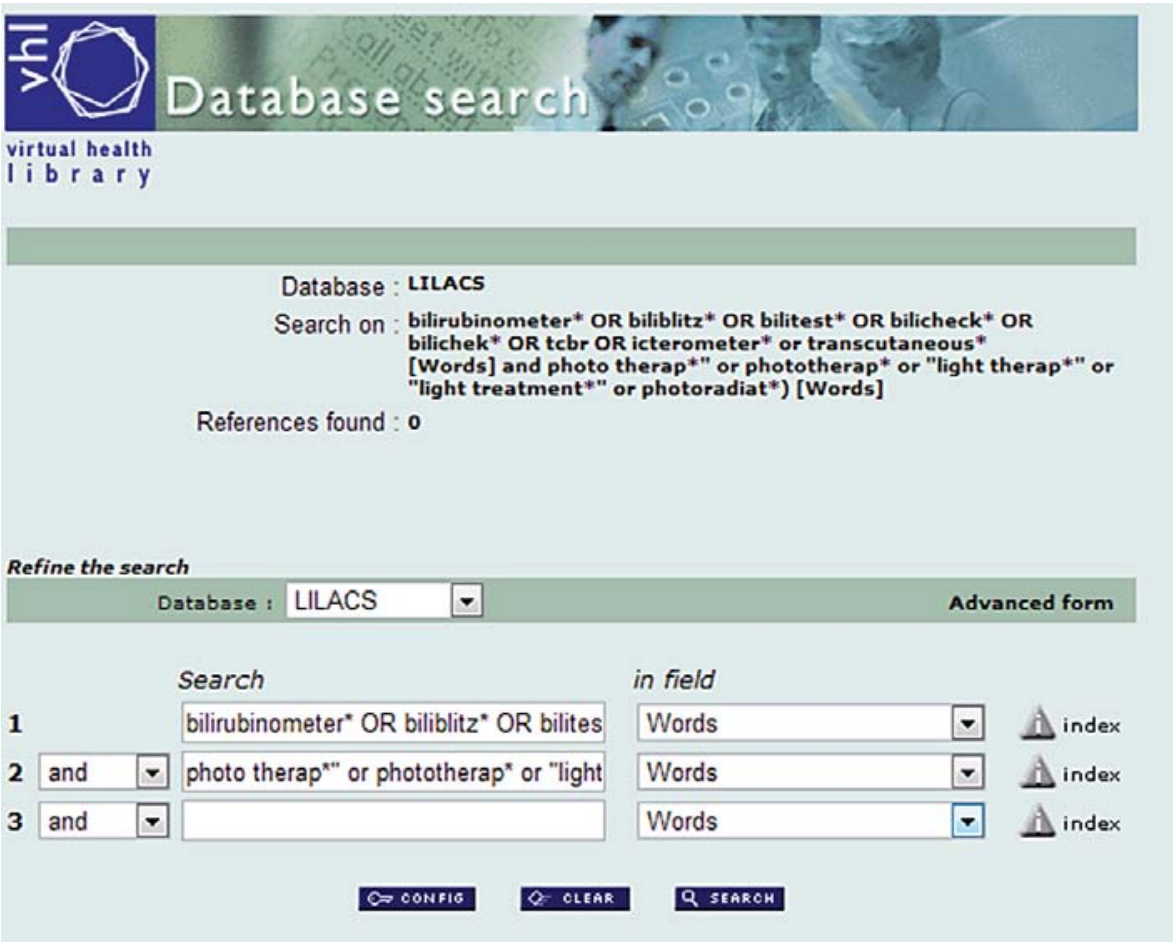

Search Updated May 8, 2014. All databases plus PubMed 38 record from 2013 to current.

Reliability of Transcutaneous Bilirubin Devices
Neonatology 2016;109:203-212

DOI: $10.1159 / 000442195$ 


\section{References}

1 Bhutani VK, Stark AR, Lazzeroni LC, Poland R, Gourley GR, Kazmierczak S, et al: Predischarge screening for severe neonatal hyperbilirubinemia identifies infants who need phototherapy. J Pediatr 2013;162:477-482.

2 Keren R, Tremont K, Luan X, Cnaan A: Visual assessment of jaundice in term and late preterm infants. Arch Dis Child Fetal Neonatal Ed 2009;94:F317-F322.

3 Sarici SU, Serdar MA, Korkmaz A, Erdem G, Oran O, Tekinalp G, et al: Incidence, course, and prediction of hyperbilirubinemia in nearterm and term newborns. Pediatrics 2004; 113:775-780.

4 Kramer LI: Advancement of dermal icterus in the jaundiced newborn. Am J Dis Child 1969; 118:454-458.

5 Moyer VA, Ahn C, Sneed S: Accuracy of clinical judgment in neonatal jaundice. Arch Pediatr Adolesc Med 2000;154:391-394.

6 Szabo P, Wolf M, Bucher HU, Fauchere JC, Haensse D, Arlettaz R: Detection of hyperbilirubinaemia in jaundiced full-term neonates by eye or by bilirubinometer? Eur J Pediatr 2004;163:722-727.

7 Anand KJ: Pain, plasticity, and premature birth: a prescription for permanent suffering? Nat Med 2000;6:971-973.

8 El-Beshbishi SN, Shattuck KE, Mohammad AA, Petersen JR: Hyperbilirubinemia and transcutaneous bilirubinometry. Clin Chem 2009;55:1280-1287.

9 Bhutani VK, Gourley GR, Adler S, Kreamer B, Dalin C, Johnson LH: Noninvasive measurement of total serum bilirubin in a multiracial predischarge newborn population to assess the risk of severe hyperbilirubinemia. Pediatrics 2000;106:E17.

10 Teran CG, Mohamed T, Casey J: Transcutaneous bilirubinometry: comparison of two multiwavelength devices in healthy term newborns. Eur J Pediatr 2011;170:1485.

11 Fouzas S, Karatza AA, Skylogianni E, Mantagou L, Varvarigou A: Transcutaneous bilirubin levels in late preterm neonates. J Pediatr 2010;157:762-766.

12 Maisels MJ, Ostrea EM Jr, Touch S, Clune SE, Cepeda E, Kring E, et al: Evaluation of a new transcutaneous bilirubinometer. Pediatrics 2004;113:1628-1635.

13 Rubaltelli FF, Gourley GR, Loskamp N, Modi $\mathrm{N}$, Roth-Kleiner M, Sender A, et al: Transcutaneous bilirubin measurement: a multicenter evaluation of a new device. Pediatrics 2001;107:1264-1271.

14 Nagar G, Vandermeer B, Campbell S, Kumar M: Reliability of transcutaneous bilirubin devices in preterm infants: a systematic review. Pediatrics 2013;132:871-881.
15 American Academy of Pediatrics Subcommittee on Hyperbilirubinemia: Management of hyperbilirubinemia in the newborn infant 35 or more weeks of gestation. Pediatrics 2004;114:297-316.

16 Jackson CL, Tudehope D, Willis L, Law T, Venz J: Home phototherapy for neonatal jaundice - technology and teamwork meeting consumer and service need. Aust Health Rev 2000;23:162-168.

17 Reyes CA, Stednitz DR, Hahn C, Mutchie KD, McCullough SR, Kronberg K: Evaluation of the BiliChek being used on hyperbilirubinemic newborns undergoing home phototherapy. Arch Pathol Lab Med 2008;132:684-689.

18 Walls M, Wright A, Fowlie P, Irvine L, Hume R: Home phototherapy in the United Kingdom. Arch Dis Child Fetal Neonatal Ed 2004; 89:F282.

19 Zainab K, Adlina S: Effectiveness of home versus hospital phototherapy for term infants with uncomplicated hyperbilirubinemia: a pilot study in Pahang, Malaysia. Med J Malaysia 2004;59:395-401.

20 Bland JM, Altman DG: Statistical methods for assessing agreement between two methods of clinical measurement. Lancet 1986;i:307-310.

21 Whiting PF, Rutjes AW, Westwood ME, Mallett S, Deeks JJ, Reitsma JB, et al: QUADAS-2: a revised tool for the quality assessment of diagnostic accuracy studies. Ann Intern Med 2011;155:529-536.

22 Peyton PJ, Chong SW: Minimally invasive measurement of cardiac output during surgery and critical care: a meta-analysis of accuracy and precision. Anesthesiology 2010; 113:1220-1235.

23 Rylance S, Yan J, Molyneux E: Can transcutaneous bilirubinometry safely guide phototherapy treatment of neonatal jaundice in Malawi? Paediatr Int Child Health 2014;34: 101-107.

24 Dominguez OF, Ormazabal Ramos JC, Martin ZM, Domenech ME: Transcutaneous bilirubinometry: correlation of the reading site obtained with spectrophotometry and diazoreaction technique (in Spanish). An Esp Pediatr 1993;39:438-440.

25 Felc Z: Improvement of conventional transcutaneous bilirubinometry results in term newborn infants. Am J Perinatol 2005;22: 173-179.

26 Fok TF, Lau SP, Hui CW, Fung KP, Wan CW: Transcutaneous bilirubinometer: its use in Chinese term infants and the effect of haematocrit and phototherapy on the TcB index. Aust Paediatr J 1986;22:107-109.
27 Galletto P, Vignolo LC, Farina D, Gavinelli R: Supervision of neonatal jaundice by use of the transcutaneous bilirubinometer (in Italian). Minerva Pediatr 1983;35:51-55.

28 Hannemann RE, Schreiner RL, DeWitt DP, Norris SA, Glick MR: Evaluation of the Minolta bilirubin meter as a screening device in white and black infants. Pediatrics 1982;69: 107-109.

29 Kumar A, Faridi MM, Singh N, Ahmad SH: Transcutaneous bilirubinometry in the management of bilirubinemia in term neonates. Indian J Med Res 1994;99:227-230.

30 Mahajan G, Kaushal RK, Sankhyan N, Sharma RL, Nakra M: Transcutaneous bilirubinometer in assessment of neonatal jaundice in northern India. Indian Pediatr 2005;42:4145.

31 Rey Galan C, Rianco Galan I, Blanco Joglar J, Gonzalez Mencia G, Enguix Armada A: Clinical application of transcutaneous bilirubin in newborns. Rev Esp Pediatr 1992;48:296-299.

32 Tan KL, Dong F: Transcutaneous bilirubinometry during and after phototherapy. Acta Paediatr 2003;92:327-331.

33 Vaisman S, Hinrichsen M: Clinical trial of a transcutaneous bilirubinometer (in Spanish). Rev Chil Pediatr 1983;54:83-86.

34 Juster-Reicher A, Flidel-Rimon O, Rozin I, Shinwell ES: Correlation of transcutaneous bilirubinometry $(\mathrm{TcB})$ and total serum bilirubin (TsB) levels after phototherapy. J Matern Fetal Neonatal Med 2015;28:1329-1331.

35 Panburana J, Boonkasidach S, Rearkyai S: Accuracy of transcutaneous bilirubinometry compared to total serum bilirubin measurement. J Med Assoc Thai 2010;93(suppl 2): S81-S86.

36 Rehak NN, Cecco SA, Hortin GL: Photolysis of bilirubin in serum specimens exposed to room lighting. Clin Chim Acta 2008;387:181183

37 Taylor R: Interpretation of the correlation coefficient: a basic review. J Diagn Med Sonogr 1990;6:35-39.

38 Grohmann K, Roser M, Rolinski B, Kadow I, Müller C, Goerlach-Graw A, et al: Bilirubin measurement for neonates: comparison of 9 frequently used methods. Pediatrics 2006; 117:1174-1183.

39 Maisels MJ, Kring E: Transcutaneous bilirubinometry decreases the need for serum bilirubin measurements and saves money. Pediatrics 1997;99:599-601.

40 Dechartres A, Trinquart L, Boutron I, Ravaud P: Influence of trial sample size on treatment effect estimates: meta-epidemiological study. BMJ 2013;346:f2304. 